\title{
Particle Distributions in a Block Copolymer Nanocomposite
}

\author{
M. W. Matsen*, ${ }^{*}$ and R. B. Thompson \\ Department of Mathematics, University of Reading, Whiteknights, Reading, RG6 6AX, England, and \\ Department of Physics and Astronomy, University of Waterloo, Waterloo, Ontario N2L 3G1, Canada
}

Received November 6, 2007; Revised Manuscript Received January 7, 2008

\begin{abstract}
Self-consistent-field theory is used to predict the center-of-mass distribution of spherical nanoparticles embedded in the lamellar phase of a diblock copolymer melt. The calculation is performed in the dilute limit, where the particle-particle interactions have a negligible effect on the distribution. We investigate how the distribution is affected by particle radius $R$, surface affinity $\Lambda N$, diblock segregation $\chi N$, diblock composition $f$, and invariant polymerization index $\bar{N}$. While the preferred location of the particles (i.e., interface or domain center) is primarily controlled by $\Lambda N$, the degree of particle localization depends on several factors. Larger values of $R, \chi N$, and $\bar{N}$ all tend to produce narrower particle distributions.
\end{abstract}

\section{Introduction}

Conventional plastics form a wide variety of durable and lightweight materials that are easy and inexpensive to process, but they do lack many of the properties common to inorganic materials such as electrical conductivity, mechanical strength, heat resistance, magnetism, high dielectric constants, and so on. This has motivated the field of nanocomposites, where nanosized inorganic particles are dispersed in a polymer matrix, promising wonderful opportunities for material design. The latest development has been the replacement of the homopolymers by block copolymers, which self-assemble to form periodically ordered morphologies. ${ }^{1}$ In this way, the nanostructured block copolymer domains act as a scaffold that directs not only the position of the inorganic particles but also their orientation. This added sophistication in the tailoring of nanocomposites has attracted tremendous attention during the past few years, but there still remain many unresolved issues.

Hamdoun et al. ${ }^{2,3}$ were the first to create block copolymer nanocomposites by dispersing iron oxide particles of diameter $3.5 \mathrm{~nm}$ into a symmetric polystyrene-poly(butyl methacrylate) diblock copolymer matrix. They were able to position the particles in the polystyrene domains by cleverly grafting short chemically identical ligands to the nanoparticles. Further studies by Lauter-Pasyuk et al. ${ }^{4}$ examined the distribution of the particles with reflectivity experiments. They concluded that the position was largely controlled by the ratio of particle diameter to domain size, with the larger particles favoring the domain centers and the small particles favoring the interfaces. This conclusion was supported by experiments of Bockstaller et al., ${ }^{5}$ but subsequent studies by Bockstaller and Thomas ${ }^{6}$ and by Chiu et al. ${ }^{7}$ observed counterexamples indicating that the situation is rather more complicated. Progress is now continuing with more systematic experiments by Kramer and co-workers, ${ }^{7-10}$ where the surface affinity of the particles is finely tuned by coating them with either a mixture of ligands or random-copolymer ligands. Furthermore, these newer experiments have measured the particle distributions with much greater sensitivity using electron microscopy. In addition to particle location, experiments are also investigating other important issues such as the critical loading beyond which the particles macrophase separate, ${ }^{1,8}$ the

\footnotetext{
* Corresponding author. E-mail: m.w.matsen@reading.ac.uk

University of Reading.

$\doteqdot$ University of Waterloo.
}

effect of particles on the block copolymer period, ${ }^{2,8}$ and particleinduced phase transitions of the block copolymer morphology. $1,8,10-12$

In principle, the statistical mechanics of a nanocomposite is a simple extension to that of an ordinary block copolymer melt. ${ }^{13}$ For the simple $\mathrm{A}-\mathrm{B}$ diblock architecture, the partition function generalizes to

$$
Z \propto \int \prod_{\alpha=1}^{n_{\mathrm{d}}} \tilde{D} \mathbf{r}_{\alpha} \prod_{\beta=1}^{n_{\mathrm{p}}} \mathrm{d} \mathbf{R}_{\beta} \exp \left(-\hat{U} / k_{\mathrm{B}} T\right) \delta\left[\hat{\phi}_{\mathrm{A}}+\hat{\phi}_{\mathrm{B}}+\hat{\phi}_{\mathrm{P}}-1\right]
$$

where in addition to the usual integrals over the configurations, $\mathbf{r}_{\alpha}(s)$, of the $n_{\mathrm{d}}$ polymers there are also integrals over the centerof-mass positions, $\mathbf{R}_{\beta}$, of the $n_{\mathrm{p}}$ particles. (Note that $s$ is a parameter that runs from 0 to 1 along the backbone of each polymer, and the particles are assumed to be spherical such that they have no orientational degrees of freedom.) Naturally, the interaction energy, $\hat{U}$, must be generalized to include the additional interactions between the polymers and the particles. The excluded-volume interactions are left out of $\hat{U}$, as they are accounted for by the Dirac delta functional, which selects those states where the dimensionless A segment, B segment, and particle concentrations add up to one. In the absence of particles, the excluded-volume interactions between the polymers can be treated in the mean-field approximation as done by the very successful self-consistent-field theory (SCFT). ${ }^{13}$ However, the same is not true of solid particles, where it is common practice to implement density-functional theory (DFT) to account for their strong excluded-volume interactions. In this spirit, Thompson and co-workers ${ }^{14}$ combined SCFT with DFT to produce a hybrid theory, which is now widely used to study the various aspects of nanocomposites. ${ }^{15,16}$ Although the SCFT/DFT approach is numerically efficient and capable of examining the triply periodic morphologies exhibited by these systems, it still only treats the excluded-volume interactions between the polymers and the particles in the mean-field approximation.

Of course, simulations ${ }^{17,18}$ provide one way around this problem, but the high computational cost restricts their application to short polymers. Sides et al. ${ }^{11}$ have outlined another approach that also overcomes the problem, which involves 


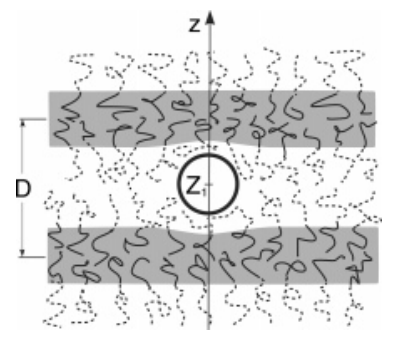

Figure 1. Schematic diagram of a spherical nanoparticle positioned at $Z_{1}$ along the $z$ axis in a diblock copolymer lamellar morphology of period, $D$. Note that the $z$ axis runs through the center of the particle and normal to the lamellae, such that it corresponds to an axis of rotational symmetry.

separating the statistical mechanics into two steps. First, a partial partition function

$$
Z\left[\left\{\mathbf{R}_{\beta}\right\}\right] \propto \int \prod_{\alpha=1}^{n_{\mathrm{d}}} \tilde{D} \mathbf{r}_{\alpha} \exp \left(-\hat{U} / k_{\mathrm{B}} T\right) \delta\left[\hat{\phi}_{\mathrm{A}}+\hat{\phi}_{\mathrm{B}}+\hat{\phi}_{\mathrm{P}}-1\right]
$$

is calculated for a fixed distribution, $\left\{\mathbf{R}_{\beta}\right\}$, of particles. Because the particles are fixed, this step can now be performed using SCFT without the polymers violating their excluded volume. Furthermore, the SCFT should provide accurate predictions so long as the block copolymer melt is well segregated (i.e., the A- and B-rich domains are relatively pure). ${ }^{19}$ Next the full partition function

$$
Z \propto \int \prod_{\beta=1}^{n_{\mathrm{p}}} \mathrm{d} \mathbf{R}_{\beta} Z\left[\left\{\mathbf{R}_{\beta}\right\}\right]
$$

is evaluated by integrating over the particle coordinates. Sides et al. ${ }^{11}$ performed this last step of the statistical mechanics using Brownian dynamics (BD). However, this approach is also very computational as each time step in the simulation requires a full SCFT calculation. To make the method tractable, Side et al. had to limit themselves to two dimensions and still the SCFT had to be solved on a relatively course mesh of $72 \times 80$, undoubtedly resulting in considerable numerical inaccuracy. Nevertheless, the general behavior of their simulation was in nice qualitative agreement with experiment.

Here we examine the particle distribution using a similar hybrid approach, where the integration over the polymer coordinates in eq 2 is performed using SCFT, but where the integration over the particle coordinates in eq 3 is evaluated in the ideal-gas limit. The latter limit assumes that particles are sufficiently dilute that the particle-particle interactions can be ignored, in which case the equilibrium particle distribution can be evaluated by considering an isolated particle in the effective periodic potential created by its interactions with the block copolymer matrix. Our calculation is done for the most common experimental situation of spherical nanoparticles in a block copolymer lamellar phase, as depicted in Figure 1. This simple geometry has an axial symmetry about the $z$ axis, which greatly reduces the computational cost. Although we cannot consider the same concentration of particles as Sides et al., ${ }^{11}$ we are now able to treat true three-dimensional spherical particles, and we can do so with high numerical precision.

\section{Theory}

This section outlines how the present theoretical technique differs from the SCFT/DFT approach of Thompson et al. ${ }^{14}$ As before, the polymers are modeled as Gaussian chains with a natural end-to-end length of $a N^{1 / 2}$ and a physical volume of $N / \rho_{0}$, where $N$ is the degree of polymerization, $a$ is the statistical segment length, and $\rho_{0}$ is the melt segment density. The dimensionless concentrations of $\mathrm{A}$ and $\mathrm{B}$ segments are still specified by

$$
\begin{aligned}
& \hat{\phi}_{\mathrm{A}}(\mathbf{r})=\frac{N}{\rho_{0}} \sum_{\alpha=1}^{n_{\mathrm{d}}} \int_{0}^{f} \mathrm{~d} s \delta\left(\mathbf{r}-\mathbf{r}_{\alpha}(s)\right) \\
& \hat{\phi}_{\mathrm{B}}(\mathbf{r})=\frac{N}{\rho_{0}} \sum_{\alpha=1}^{n_{\mathrm{d}}} \int_{f}^{1} \mathrm{~d} s \delta\left(\mathbf{r}-\mathbf{r}_{\alpha}(s)\right)
\end{aligned}
$$

respectively, where $f$ denotes the fraction of the diblock formed by A segments. Again, the particles have a center-of-mass distribution of

$$
\hat{\rho}_{\mathrm{P}}(\mathbf{r})=\sum_{\beta=1}^{n_{\mathrm{p}}} \delta\left(\mathbf{r}-\mathbf{R}_{\beta}\right)
$$

but now their dimensionless concentration is calculated as

$$
\hat{\phi}_{\mathrm{P}}(\mathbf{r})=\int \mathrm{d} \mathbf{r}^{\prime} P\left(\left|\mathbf{r}^{\prime}\right|\right) \hat{\rho}_{\mathrm{P}}\left(\mathbf{r}+\mathbf{r}^{\prime}\right)
$$

where

$$
P(r) \equiv \frac{1}{2}\left[1+\tanh \left(\frac{2(R-r)}{w_{\mathrm{p}}}\right)\right]
$$

defines the particle profile. Reference 14 assumed a step profile where $P(r)$ switches from 1 to 0 at the particle radius $R$, whereas we now assume a gradual interface with a characteristic width of $w_{\mathrm{p}}$. This is done partly out of numerical necessity as we will discuss later and partly to account for the penetrability of the short polymer ligands routinely grafted to the particles in order to control their surface affinity. The interaction between $\mathrm{A}$ segments, B segments, and particles is now defined by

$$
\frac{\hat{U}}{k_{\mathrm{B}} T}=\rho_{0} \int \mathrm{d} \mathbf{r}\left[\chi \hat{\phi}_{\mathrm{A}} \hat{\phi}_{\mathrm{B}}-\frac{4 \Lambda}{w_{\mathrm{p}}} \hat{\phi}_{\mathrm{P}}\left(\hat{\phi}_{\mathrm{A}}-\hat{\phi}_{\mathrm{B}}\right)\right]
$$

where $\chi$ is the standard Flory-Huggins $\mathrm{A}-\mathrm{B}$ segment interaction parameter and $\Lambda$ gives the surface affinity of the particles. The latter is defined such that the interaction energy experienced by a single particle is $-\Lambda 4 \pi R^{2} k_{\mathrm{B}} T$ when immersed in $\mathrm{A}$ segments and $\Lambda 4 \pi R^{2} k_{\mathrm{B}} T$ when in contact with pure B, assuming that $w_{\mathrm{p}} \ll R$.

The SCFT remains much the same as in ref 14, except that the polymers now interact with the instantaneous particle concentration. This is reflected in the fact that the fields acting on the $\mathrm{A}$ and $\mathrm{B}$ segments

$$
\begin{aligned}
& w_{\mathrm{A}}(\mathbf{r})=\chi N \phi_{\mathrm{B}}(\mathbf{r})-\frac{4 \Lambda N}{w_{\mathrm{p}}} \hat{\phi}_{\mathrm{P}}(\mathbf{r})+\xi(\mathbf{r}) \\
& w_{\mathrm{B}}(\mathbf{r})=\chi N \phi_{\mathrm{A}}(\mathbf{r})+\frac{4 \Lambda N}{w_{\mathrm{p}}} \hat{\phi}_{\mathrm{P}}(\mathbf{r})+\xi(\mathbf{r})
\end{aligned}
$$

and the incompressibility condition

$$
\phi_{\mathrm{A}}(\mathbf{r})+\phi_{\mathrm{B}}(\mathbf{r})=1-\hat{\phi}_{\mathrm{P}}(\mathbf{r})
$$

involve $\hat{\phi}_{\mathrm{P}}(\mathbf{r})$ rather than its thermodynamic average, $\phi_{\mathrm{P}}(\mathbf{r}) \equiv$ $\left\langle\hat{\phi}_{\mathrm{P}}(\mathbf{r})\right\rangle$. Once the fields have been adjusted to satisfy these 
conditions, the effective Hamiltonian of the particles is given by

$$
\begin{array}{r}
\frac{F\left[\left\{\mathbf{R}_{\beta}\right\}\right]}{k_{\mathrm{B}} T}=-n_{\mathrm{d}} \ln Q+\frac{\rho_{0}}{N} \int \mathrm{d} \mathbf{r}\left[\chi N \phi_{\mathrm{A}} \phi_{\mathrm{B}}-\frac{4 \Lambda N}{w_{\mathrm{p}}} \hat{\phi}_{\mathrm{P}}\left(\phi_{\mathrm{A}}-\right.\right. \\
\left.\left.\phi_{\mathrm{B}}\right)-w_{\mathrm{A}} \phi_{\mathrm{A}}-w_{\mathrm{B}} \phi_{\mathrm{B}}\right]
\end{array}
$$

where $Q$ is the partition function of a single diblock molecule subject to the fields, $w_{\mathrm{A}}(\mathbf{r})$ and $w_{\mathrm{B}}(\mathbf{r})$. The statistical mechanics of the particles is then evaluated according to eq 2 , where the different particle configurations, $\left\{\mathbf{R}_{\beta}\right\}$, are weighted by $Z\left[\left\{\mathbf{R}_{\beta}\right\}\right]$ $\equiv \exp \left(-F\left[\left\{\mathbf{R}_{\beta}\right\}\right] / k_{\mathrm{B}} T\right)$.

Here we consider well-segregated symmetric diblock copolymers (i.e., $f \approx 0.5$ ), for which the preferred morphology is the simple lamellar phase. ${ }^{13}$ Furthermore, we assume that the particle concentration is sufficiently dilute that we can ignore its effect on either the symmetry or the period, $D$, of the block copolymer morphology. As mentioned previously, the low concentration allows the statistical mechanics of the particles to be done in the ideal-gas limit, where the particles can be treated in isolation (i.e., $n_{\mathrm{p}}=1$ ). This imparts an axial symmetry to the problem as illustrated in Figure 1, which we take advantage of by solving the SCFT in a cylindrical cell with reflecting boundaries on all surfaces. This boundary condition forces the top and bottom surfaces of the cell to each coincide with the midplane of a lamellar domain, which implies that the height of the cell must be an integer or half-integer number of periods. The height and diameter of the cell must also be larger than the range of the perturbation caused by the particle on the lamellar morphology; we choose a height of $3.5 \mathrm{D}$ and a diameter of $4 a N^{1 / 2}$, which provides enough volume $\left(V=14 \pi D a^{2} N\right)$ to avoid any significant finite-size effects for the parameter conditions considered in our particular study. The diffusion equation ${ }^{14}$ used by SCFT to work out the segment concentrations is solved on a discrete mesh using the standard CrankNicholson algorithm. We avoid any significant numerical inaccuracy by using 200 steps along the polymer contours and selecting a fine mesh with 400 points along the diameter and 600 points in the $z$ direction. Integrations are done by a simple trapezoidal rule, which conserves the polymer concentration accurately. The self-consistent-field eqs 10-12 are solved by the Anderson mixing technique. ${ }^{20}$

\section{Results}

We now begin by examining relatively large particles $(R=$ $0.4 a N^{1 / 2}$ and $\left.w_{\mathrm{p}}=0.15 a N^{1 / 2}\right)$ immersed in a symmetric lamellar phase $(\chi N=25$ and $f=0.5)$ with an equilibrium period of $D$ $=1.75 a N^{1 / 2}$. Figure $2 \mathrm{a}$ shows the change in free energy, $\Delta F\left(Z_{1}\right)$ $\equiv F\left(Z_{1}\right)-F(0)$, as particles of various selectivity move from the middle of a B-rich domain $\left(Z_{1}=-0.25 D\right)$ through an interface $\left(Z_{1}=0\right)$ past the center of the neighboring A-rich domain $\left(Z_{1}=0.25 D\right)$ and on to the next interface $\left(Z_{1}=0.5 D\right)$. The average force exerted on a particle by the block copolymer is given by minus the free energy gradient, and thus a neutral particle $(\Lambda N=0)$ tends to be pushed toward the interfaces. The resulting center-of-mass distribution, $\rho_{\mathrm{P}}(z)$, is plotted in Figure $2 b$ for diblock copolymers of various invariant polymerization indices, $\bar{N} \equiv \rho_{0}^{2} a^{6} N$, spanning a full range of experimentally relevant values. ${ }^{21}$ The finite width of $\rho_{\mathrm{P}}(z)$ can be attributed to thermal fluctuations, which is why it narrows with increasing molecular weight. ${ }^{22}$ As a particle develops an affinity for A segments (i.e., $\Lambda N>0$ ), the free energy minima shift from the interfaces to inside the A-rich domains. Figure


Figure 2. (a) Relative free energy, $\Delta F\left(Z_{1}\right) \equiv F\left(Z_{1}\right)-F(0)$, as a function of particle position, $Z_{1}$, for particles with various surface affinities, $\Lambda N$. The average center-of-mass distribution, $\rho_{\mathrm{P}}(z)$, is plotted below for (b) neutral particles and (c) particles with an affinity for the A-rich domain. The unperturbed block copolymer interfaces occur at $z=0$ and $0.5 D$, and the center of the A-domain is at $z=0.25 D$, where $D$ is the lamellar period. All calculations are done for a fixed particle radius of $R=0.4 a N^{1 / 2}$ and the same symmetric lamellar morphology of $\chi N=25$ and $f=0.5$.

2c plots $\rho_{\mathrm{P}}(z)$ for the high selectivity of $\Lambda N=0.6$, where the preferred locations of the particle are at the very center of the A domains.

Figure 3 illustrates the response of the block copolymer morphology as a neutral $R=0.4 a N^{1 / 2}$ particle moves through it. When the particle is centered on an interface (e.g., $Z_{1}=0$ ), its perturbation on the morphology is remarkably small. However, as $Z_{1}$ increases and the particle moves away from its preferred location, the interface is dragged along with the particle so as to minimize the increase in interfacial area. Because of the natural tendency for the domains to maintain a uniform thickness, ${ }^{19}$ this perturbation also has a significant effect on the neighboring interfaces. Nevertheless, the morphology evolves continuously until $Z_{1}=0.1947 \mathrm{D}$, at which point the $z=0.5 \mathrm{D}$ interface jumps into contact with the particle, as demonstrated in Figures 3c,d. The particle maintains contact with the two interfaces up to the point, $Z_{1}=0.3053 D$, where the $z=0$ interface then suddenly detaches. These abrupt transformations in the morphology, denoted by solid dots in Figure 2, are firstorder transitions that cause discontinuities in the force (i.e., free energy gradient).

Figure 4 shows the effect of a selective particle $(\Lambda N=0.6)$ as it moves through the lamellar morphology. When the particle 

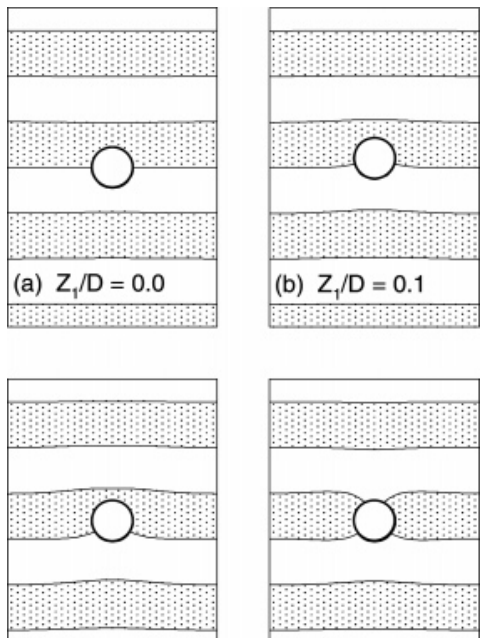

(c) $Z_{1} / D=0.1947$

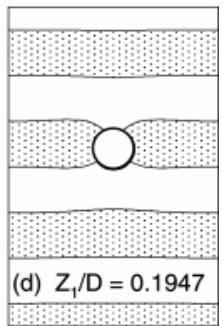

Figure 3. Block copolymer morphology of $\chi N=25$ and $f=0.5$ with a neutral, $\Lambda N=0$, particle of radius $R=0.4 a N^{1 / 2}$ located at various positions, $Z_{1}$.



(a) $Z_{1} / D=-0.1256$


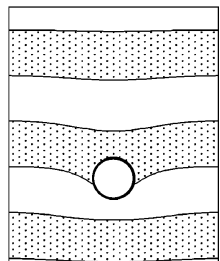

(b) $Z_{1} / D=-0.1256$

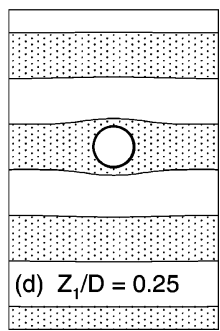

Figure 4. Analogous plots to those in Figure 3, but for a particle with an affinity, $\Lambda N=0.6$, for the shaded A-rich domains.

is near the middle of the $\mathrm{B}$ domain (e.g., $Z_{1} \approx-0.25 D$ ), its affinity for A segments pulls the two neighboring A domains into contact. As the particle is moved toward, for example, the upper $z=0$ interface, it ultimately breaks contact with the lower $z=-0.5 D$ interface at $Z_{1}=-0.1256 D$, causing a discontinuous change in the morphology as illustrated by Figures 4a,b. When this happens, there is an abrupt increase in the force pulling the particle toward the center of the A domain. The force results from the deformation of the A domain, which engulfs the particle so as to shield it from the B segments (see Figure 4c). The domain shape relaxes as the particle moves toward the center of the domain $\left(Z_{1}=0.25 D\right)$ and the force vanishes, but a significant bulge remains because of the relatively large size of the particle (see Figure 4d).

Although there are experiments ${ }^{23}$ where the particle diameter is similar in size to the domains, the vast majority of experiments $2,3,5-10$ involve particles that are a fraction of the domain size. Therefore, we turn our attention to smaller particles of radius, $R=0.2 a N^{1 / 2}$, but we still keep the width of their profile as $w_{\mathrm{p}}=0.15 a N^{1 / 2}$. The free energy curves are shown in Figure 5a for equivalent conditions to those of the larger particle. A neutral particle still maintains a significant preference for the
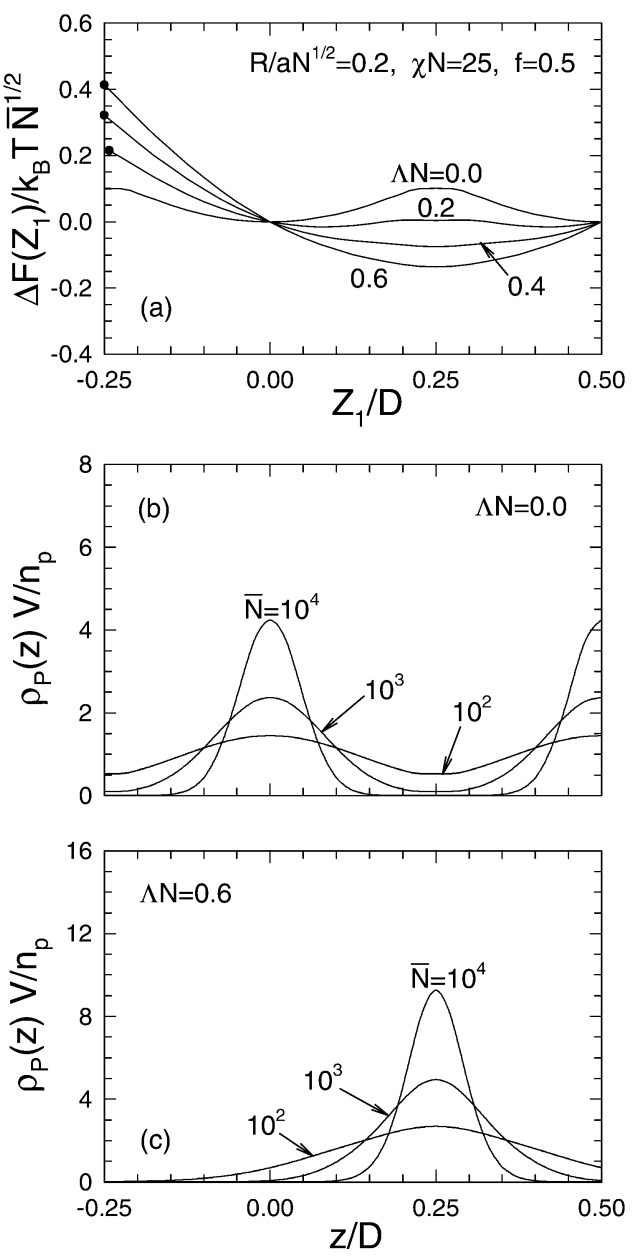

Figure 5. Analogous plots to those in Figure 2, but for a particle of smaller radius, $R=0.2 a N^{1 / 2}$.

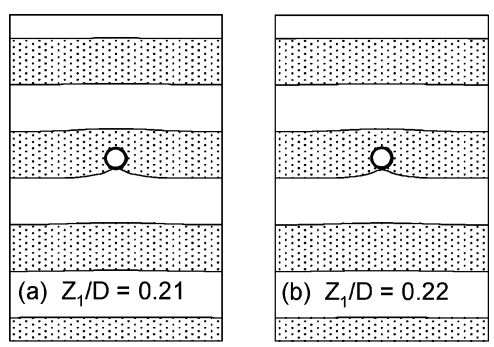

Figure 6. Images of a neutral, $\Lambda N=0$, particle of radius $R=0.2 a N^{1 / 2}$ gradually detaching from the interface of a $\chi N=25$ and $f=0.5$ diblock copolymer morphology.

interfaces, although a somewhat weaker one than for the larger particle as evident by comparing Figures $2 \mathrm{~b}$ and $5 \mathrm{~b}$. Likewise, a positive surface affinity does not cause the same degree of localization within the A domains as illustrated by comparing Figures $2 \mathrm{c}$ and $5 \mathrm{c}$.

Many of the morphological transitions that occurred for the larger particle are no longer present for the smaller particle. The small neutral particle, for example, passes from domain to domain without any sudden changes in the morphology as illustrated in Figure 6. However, there are still discontinuous transitions, marked by solid dots in Figure 5a, as highly selective particles pass through the unfavorable domain. Take the case of a particle in the B domain with an affinity of $\Lambda N=0.6$ for A segments. Because of the small particle size, it is unable to simultaneously attract both of the neighboring A domains, but the interaction is still strong enough to attract the closest of the two interfaces as illustrated in Figure $7 b$. Consequently, a phase 

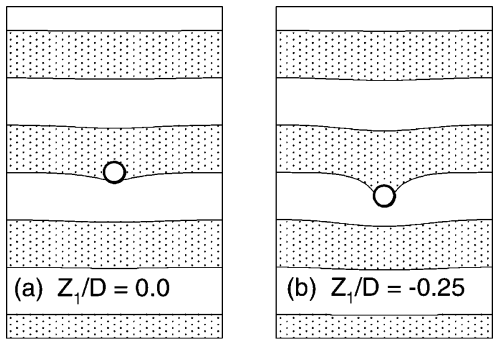

Figure 7. Images of a strongly selective, $\Lambda N=0.6$, particle of radius $R=0.2 a N^{1 / 2}$ as it is pulled out of its preferred A-rich (shaded) domain.
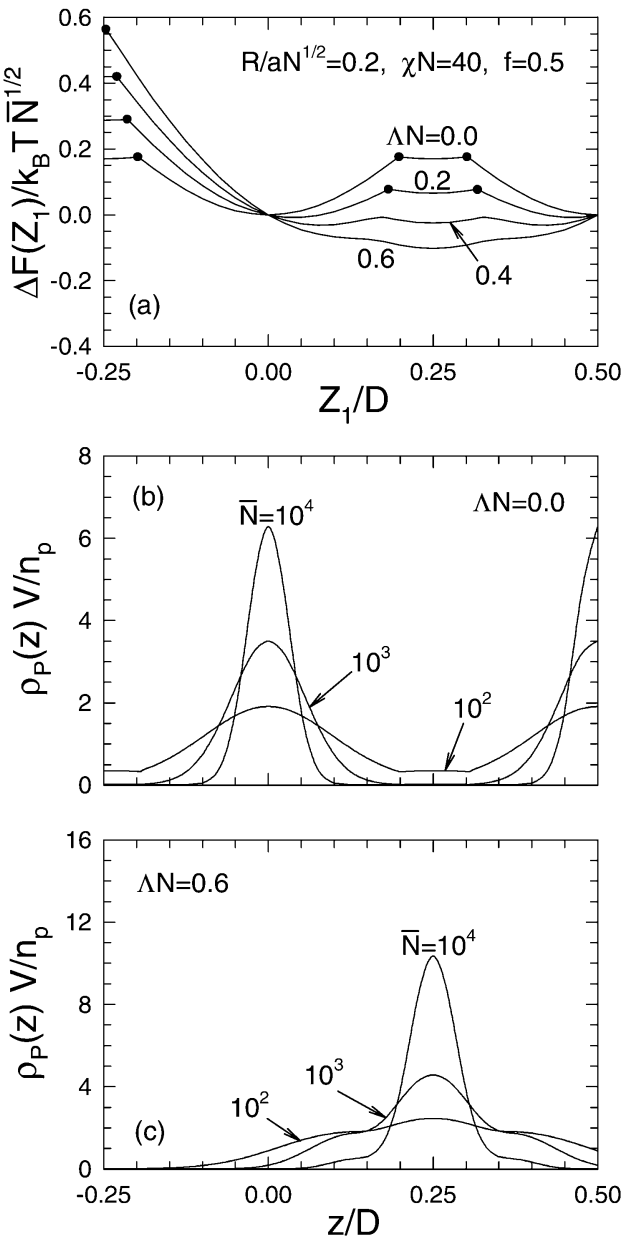

Figure 8. Analogous plots to those in Figure 5, but for a more strongly segregated morphology of $\chi N=40$.

transition will occur as the particle crosses the center of the B domain $\left(Z_{1}=-0.25 D\right)$ and the contact switches from one interface to the other.

We now repeat our calculation for a strongly segregated block copolymer melt of $\chi N=40$, where the increased interfacial tension swells the domain spacing to $D=1.95 a N^{1 / 2}$. Figure 8 shows the variation in free energy as $R=0.2 a N^{1 / 2}$ particles of various surface affinity are moved through the morphology. Because of the higher interfacial tension, there is a greater tendency for the particles to reside at the interface in order to shield the unfavorable contact between A and B segments. Not only are the neutral particles much more localized at the interface as illustrated in Figure 8b, a surface affinity of $\Lambda N=$ 0.6 is now just barely enough to overcome the attraction to the interface. This latter fact is illustrated in Figure $8 \mathrm{c}$, where the peak in $\rho_{\mathrm{P}}(z)$ at the center of the A domain is supplemented with significant shoulders next to each of the neighboring interfaces.

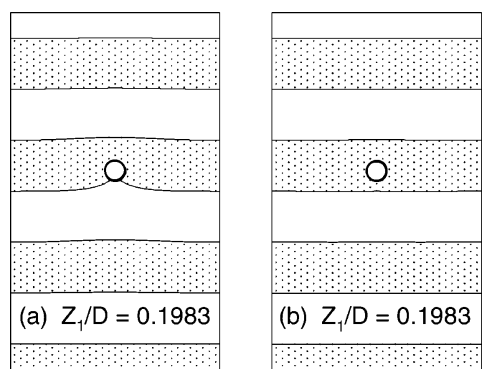

Figure 9. Analogous plots to those in Figure 6, but for a higher degree of segregation, $\chi N=40$, where the interface detaches abruptly from the particle.
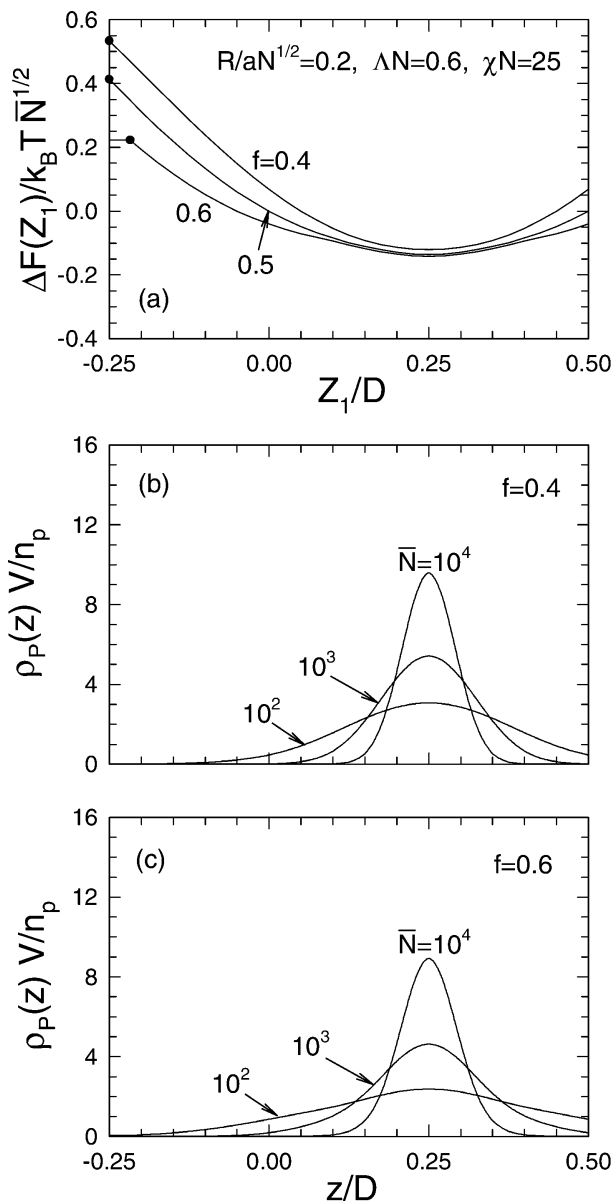

Figure 10. Analogous plots to those in Figure 5, but calculated for a range of diblock compositions, $f$, while holding the particle radius fixed at $R=0.2 a N^{1 / 2}$ and the surface affinity fixed at $\Lambda N=0.6$.

The morphological transitions that disappeared for the neutral particle with the reduction in $R$ have now reappeared with the increase in $\chi N$. This is because the distance a neutral particle is able to drag an interface is related to its cross-sectional area times the interfacial tension. Thus, when the interface does finally detach from the particle, it has built up so much energy that it snaps back to its unperturbed position with an abrupt phase transition, as demonstrated in Figure 9.

We conclude our study by examining the effect of domain size on the localization of the particles, which we accomplish by adjusting the diblock composition, $f$. (Note that the relative sizes of the $\mathrm{A}$ and $\mathrm{B}$ domains track the composition of the diblock, while the overall period, $D$, of the lamellar phase remains essentially constant. ${ }^{24}$ ) Figure 10 a shows the free energy of particles with an affinity for A segments $(\Lambda N=0.6)$ as they move through a series of lamellar morphologies with different sized A domains. The resulting particle distributions for small 
and large domains are shown in parts $\mathrm{b}$ and $\mathrm{c}$ of Figure 10, respectively. The difference is fairly minimal, but the particles are somewhat more localized in the smaller domain for $f=$ 0.4. This should be expected given that a smaller A domain implies less volume over which the particles can move without contacting a B domain.

\section{Discussion}

Our study provides the most quantitatively reliable predictions to date for the particle distributions in a block copolymer nanocomposite. Not only does it fully respect the excluded volume of the particles, the particles are modeled as true threedimensional spheres and the numerical calculations are performed with high precision. However, the predictions are restricted to low particle concentrations because the statistical mechanics over the particle coordinates, $\mathbf{R}_{\beta}$, in eq 3 are treated in the ideal-gas limit. This restriction will be most serious when the distribution, $\rho_{\mathrm{P}}(\mathbf{r})$, is narrow and the particles are essentially arranged in two-dimensional planes. For the approximation to remain valid, the total cross-sectional area of the particles, $n_{\mathrm{p}} \pi R^{2}$, must be much smaller than the total interfacial area, $2 V / D$, of the block copolymer. This requires that the particle volume fraction satisfies

$$
\bar{\phi}_{\mathrm{P}} \ll R / D
$$

For typical experimental particle sizes (e.g., $R / D \sim 0.1$ ), we expect that our predicted distributions, $\rho_{\mathrm{P}}(z)$, will be reasonably accurate for particle volume fractions of less than $1 \%$.

For these low concentrations, it is unlikely that there would be any significant deviation of the lamellar period, $D$, from that of the neat diblock copolymer melt, ${ }^{8}$ as we have assumed in our present calculation. Nevertheless, it could be interesting to calculate the leading order correction for nonzero $\bar{\phi}_{\mathrm{P} .}{ }^{17}$ Provided the particles still remain sufficiently dilute, the partition function in eq 3 can still be evaluated in the ideal-gas limit as a function of the domain size. The corrected equilibrium period would then be obtained by minimizing the resulting free energy, $F=k_{\mathrm{B}} T$ $\ln Z$.

For more concentrated particles, the shape of $\rho_{\mathrm{P}}(\mathbf{r})$ would begin to depend on $\bar{\phi}_{\mathrm{P}}{ }^{7}$ To predict the change in the distribution, one would have to go beyond the ideal-gas approximation and account for the effective particle-particle interactions by a cluster expansion or an appropriate density functional theory. Of course, this would be much more complicated than for classical gases because of the periodic background potential and the fact that the effective interaction between two particles depends on their actual positions, $Z_{1}$ and $Z_{2}$, as well as their lateral separation. Apart from the special case of when the particles are aligned in the $z$ direction, a numerically accurate calculation would be highly computational. Reister and Fredrickson ${ }^{25}$ have evaluated these effective interactions, but only for cylindrical and planar objects oriented parallel to the lamellae. As a simple approximation, one could ignore the effect of the block copolymer matrix and just include the direct hardcore interaction, which for spheres is only a function of their separation.

The virtue of the hybrid approach introduced by Sides et al. ${ }^{11}$ and used here is that it prevents the polymers from entering the excluded volume of the particles, $\hat{\phi}_{\mathrm{P}}(\mathbf{r})$. Provided that the width of the particle profile in eq 7 is narrow (i.e., $w_{\mathrm{p}} \ll a N^{1 / 2}$ ), the entropic penalty of restricting the polymer configurations is well approximated by ${ }^{13}$

$$
\frac{F_{\text {conf }}}{k_{\mathrm{B}} T}=\frac{\rho_{0} a^{2}}{24} \int \mathrm{d} \mathbf{r} \frac{\left|\nabla \hat{\phi}_{\mathrm{P}}\right|^{2}}{1-\hat{\phi}_{\mathrm{P}}}
$$

This penalty should be relatively constant, assuming that the particles remain reasonably dispersed. However, this is not the case in the SCFT/DFT approach, ${ }^{14-16}$ where the polymers are effectively excluded from the average particle positions. In that case, the entropic penalty is still approximated by eq 13 , but with $\hat{\phi}_{\mathrm{P}}(\mathbf{r})$ replaced by its thermodynamic average, $\phi_{\mathrm{P}}(\mathbf{r})$. Consequently, the entropic penalty in the SCFT/DFT calculations increases as the particles become more localized, which leads to an unphysical mechanism that broadens the particle distribution.

The violation of the excluded volume also impacts the particle-polymer interaction, $\rho_{0} \int \mathrm{d} \mathbf{r}\left[\chi_{\mathrm{AP}} \hat{\phi}_{\mathrm{A}} \hat{\phi}_{\mathrm{P}}+\chi_{\mathrm{BP}} \hat{\phi}_{\mathrm{B}} \hat{\phi}_{\mathrm{P}}\right]$, used in the SCFT/DFT approach. ${ }^{14-16}$ Given that the SCFT/DFT calculations assume a sharp particle profile with $w_{\mathrm{p}}=0$, the interaction would be identically zero if it was not for the fact that the polymers interact with the average, $\phi_{\mathrm{P}}(\mathbf{r})$, rather than the instantaneous, $\hat{\phi}_{\mathrm{P}}(\mathbf{r})$, particle concentration. One fairly obvious consequence of this shortcoming is that the polymerparticle interaction effectively shuts off as the particles become increasingly localized.

Here our particles were given a soft interface with a characteristic width of $w_{\mathrm{p}}$, rather than the step profile used in most previous studies. This was partly to account for the layer of ligands that are generally grafted to the particles in real experiments, but it was also done out of computational necessity. ${ }^{11}$ Without a finite width $w_{\mathrm{p}}$, the entropic penalty in eq 15 would diverge. This raises a serious question with ref 25 , where the particles were given a step profile. Although their real-space calculation still produces a finite free energy so long as the spacing of their mesh remains finite, there is no guarantee that their nonconverged results are quantitatively meaningful. Note that it is possible to allow a step profile, but then the particle surface has to be treated as a reflecting boundary. ${ }^{13}$

Pryamitsyn and Ganesan ${ }^{17}$ have recently performed an analogous calculation to ours, but using the strong-stretching theory (SST) of Semenov ${ }^{26}$ supplemented by a number of approximations. For instance, their calculation ignores the change in the polymer trajectories required to accommodate the particle. It simply approximates $F\left(Z_{1}\right) / k_{\mathrm{B}} T$ as an enthalpy term calculated by assuming that the block copolymer interface remains flat and a pressure term, $\xi\left(Z_{1}\right) 4 \pi R^{3} / 3$, evaluated at the center-of-mass of the particle. The merit of this SST approach is the transparency of its analytical predictions, but of course there is a cost in terms of accuracy. We can now assess the resulting inaccuracy by comparing the particle distribution plotted in their Figure 1a, for a particle radius of $R=0.204 a N^{1 / 2}$ and symmetric diblocks of $\chi N=30$ and $\bar{N}=864$, to our $\rho_{\mathrm{P}}(z)$ in Figure 5, for very similar parameters. For strong surface affinities, the SST approach predicts a much different shape for $\rho_{\mathrm{P}}(z)$ than SCFT, and for neutral particles, it gives a much narrower distribution. Evidently, the approximations involved require smaller particles and/or higher degrees of segregation. Even then, the SST calculation may seriously overestimate the localization of neutral particles by not allowing the interface to follow the motion of the particles.

We can also compare our predictions to experiment. Kramer and co-workers ${ }^{7-9}$ have examined particle distributions in symmetric PS-P2VP diblock melts for small gold spheres coated with short PS and P2VP ligands so as to control the surface affinity from highly selective to relatively neutral. In most cases, the measured distributions have a Gaussian shape 
very similar to our predicted distributions. The one exception ${ }^{8}$ is for PS selective particles, where the distribution exhibits distinct shoulders next to the maximum. It is remarkably similar in shape to our $\bar{N}=10^{3}$ distribution in Figure $8 \mathrm{c}$ for the $\chi N=$ 40 lamellar phase. This does not, however, imply that the nonGaussian distribution in ref 8 occurred because the block copolymer was highly segregated. It occurs simply because of an intermediate surface affinity relative to the block copolymer interfacial tension. After all, the surface affinity can be varied continuously, and thus there must exist non-Gaussian distributions intermediate to the Gaussian distributions of neutral and strongly selective particles.

It has been suggested, initially by Lauter-Pasyuk et al., ${ }^{4}$ that small particles tend to accumulate at the interface. We find that small particles do in general have broader distributions, but they have no particular preference for the interface. In reality, the neutron reflectivity experiments did not have the sensitivity to make definitive statements about the detailed particle distributions. On the other hand, the direct microscopy measurements by Bockstaller et al. ${ }^{5}$ clearly showed small particles at the interface and large particles in the domain centers. However, their small particles where gold while their large particles were made of silica, and furthermore the ligands on the two particles had different lengths and different grafting densities. Thus, it is impossible to make the general conclusion that smaller particles prefer the interface, since the particles differed in more than just their size.

This mistaken conclusion that small particles prefer the interface can be attributed to the fact that it is very difficult to performed controlled experiments where only a single parameter is varied. A similar situation arises in the more recent experiments of Costanzo and Beyer. ${ }^{23}$ They examined an interesting system involving gold nanoparticles grafted with short PSPEG diblock ligands in a symmetric PS-PMMA diblock morphology. The ligands were anchored to the gold particles by their PS ends exposing the PEG, which produced an affinity for the PMMA domains. When the temperature was increased, the PEG blocks would detach and the particles would then migrate toward the PS domains. Costanzo and Beyer argued that particle localization should improve by making the particles small relative to the domain size, and they supported this claim by showing improved localization in larger domains produced by increasing the molecular weight of the PS-PMMA matrix. However, comparison of Figures $2 \mathrm{~b}$ and $5 \mathrm{~b}$ indicates that larger particles are in fact more selective, which makes sense since their total surface energy is greater. Furthermore, when we increased the domain size by changing $f$, the degree of localization was slightly reduced as revealed by carefully comparing parts $\mathrm{b}$ and $\mathrm{c}$ of Figure 10. The oversight by Costanzo and Beyer is that a larger molecular weight of PS-PMMA not only increases the domain size but also increases the degree of segregation, $\chi N$, and the invariant polymerization index, $\bar{N}$, both of which improve localization according to our SCFT calculations.

In addition to the ability to vary individual parameters independently, the SCFT also offers an immense versatility, which will be important for any truly accurate modeling of the experiments. Indeed, Kramer and co-workers have suggested that theoretical calculations may need to consider details such as the presence of solvent used in casting nanocomposites. ${ }^{7,10}$ Fortunately, it is a trivial matter to extend SCFT to include solvent, although there will be the added burden of having to deal with the extra interaction parameters. ${ }^{27}$ Furthermore, they suggested that the detailed distribution of the ligand brush could be important, particularly because the mobility of the grafting points could result in Janus-type particles. ${ }^{8,9}$ Again, the SCFT is fully capable of explicitly including grafted chains rather than just assigning a gradual profile to the particle. There is one technical difficulty in that the mesh must be particularly fine next to the particle (i.e., grafting surface), but there is an efficient way of dealing with this by overlaying two separate coordinate systems. $^{28}$ As long as the axial symmetry in Figure 1 is maintained, there is considerable scope for any necessary extensions to the model.

\section{Summary}

The distribution of spherical nanoparticles in a block copolymer lamellar phase has been examined using a SCFT approach motivated by Sides et al., ${ }^{11}$ which properly respects the excluded volume of the particles unlike the previous SCFT/DFT calculations. ${ }^{14-16}$ In contrast to the earlier work in ref 11 , we consider the ideal-gas limit where the particles can be treated in isolation (see Figure 1), allowing the calculation to be applied to true three-dimensional spherical particles while maintaining high numerical precision. Our calculation is similar in nature to the SST treatment by Pryamitsyn and Ganesan, ${ }^{17}$ but our approach relaxes the assumption of strongly stretched chains, forces the polymers to deviate around the particle, and allows the interface to bend in response to the particle location. Indeed, we find that the interface has a strong tendency to follow the position of the particle and that it often detaches from the particle with an abrupt transition.

Not only does our SCFT treatment predict particle distributions much more similar to those measured by experiment, ${ }^{7-9}$ it also has the scope for further extensions. For instance, it would be a straightforward matter to include small amounts of solvent ${ }^{27}$ in order to understand how the morphology might be affected by the solvent-casting procedure used to produce the nanocomposites. Furthermore, the polymer ligands grafted to particles to control the surface interaction could be explicitly included, if need be. Although we have ignored the effect of the particles on the domain spacing, it could readily be included. It is also conceivable that the effective pairwise interactions between particles could be incorporated in order to treat higher particle loadings. However, the extension to more complex block copolymer morphologies (e.g., gyroid) would be unrealistic because of the lack of symmetry involved. Nevertheless, the simple lamellar morphology provides the ideal background for studying the general principles of these nanocomposite materials, and the present SCFT approach offers a reliable strategy for making quantitative predictions.

Acknowledgment. This work was supported by EPSRC (Grant EP/D031494/01) and by NSERC.

\section{References and Notes}

(1) Bockstaller, M. R.; Michiewicz, R. A.; Thomas, E. L. Adv. Mater. 2005, 17, 1331.

(2) Hamdoun, B.; Ausserré, D.; Cabuil, V.; Joly, S. J. Phys. II 1996, 6, 503.

(3) Hamdoun, B.; Ausserré, D.; Joly, S.; Gallot, Y.; Cabuil, V.; Clinard, C. J. Phys. II 1996, 6, 493.

(4) Lauter-Pasyuk, V.; Lauter, H. J.; Ausseré, D.; Gallot, Y.; Cabuil, V.; Kornilov, E. I.; Hamdoun, B. Physica B 1998, 1092, 241-243; Physica $B$ 1998, 248, 243.

(5) Bockstaller, M. R.; Lapetnikov, Y.; Margel, S.; Thomas, E. L. J. Am Chem. Soc. 2003, 125, 5276.

(6) Bockstaller, M. R.; Thomas, E. L. Phys. Rev. Lett. 2004, 93, 166106.

(7) Chiu, J. J.; Kim, B. J.; Yi, G.-R.; Bang, J.; Kramer, E. J.; Pine, D. J. Macromolecules 2007, 40, 3361. 
(8) Kim, B. J.; Fredrickson, G. H.; Hawker, C. J.; Kramer, E. J. Langmuir 2007, 23, 7804.

(9) Chiu, J. J.; Kim, B. J.; Kramer, E. J.; Pine, D. J. J. Am. Chem. Soc. 2005, 127, 5036 .

(10) Kim, B. J.; Chiu, J. J.; Yi, G.-R.; Pine, D. J.; Kramer, E. J. Adv. Mater 2005, 17, 2618.

(11) Sides, S. W.; Kim, B. J.; Kramer, E. J.; Fredrickson, G. H. Phys. Rev. Lett. 2006, 96, 250601.

(12) Yeh, S.-W.; Wei, K.-H.; Sun, Y.-S.; Jeng, U. S.; Liang, K.-S. Macromolecules 2005, 38, 6559.

(13) Matsen, M. W. In Soft Matter; Gompper, G., Schick, M., Eds.; WileyVCH: Weinheim, 2006; Vol. 1.

(14) Thompson, R. B.; Ginzburg, V. V.; Matsen, M. W.; Balazs, A. C Science 2001, 292, 2469; Macromolecules 2002, 35, 1060.

(15) Balazs, A. C. Curr. Opin. Solid State Mater. Sci. 2003, 7, 27.

(16) Cao, D.; Wu, J. J. Chem. Phys. 2007, 126, 144912. Zhang, L.; Lin, J.; Lin, S. Macromolecules 2007, 40, 5582.
(17) Pryamitsyn, V.; Ganesan, V. Macromolecules 206, 39, 8499.

(18) Schultz, A. J.; Hall, C. K.; Genzer, J. Macromolecules 2005, 38, 3007.

(19) Matsen, M. W. J. Phys.: Condens. Matter 2002, 14, R21.

(20) Thompson, R. B.; Rasmussen, K. Ø.; Lookman, T. J. Chem. Phys. 2004, 120, 31 .

(21) Bates, F. S.; Schulz, M. F.; Khandpur, A. K.; Förster, S.; Rosedale, J. H.; Almdal, K.; Mortensen, K. Faraday Discuss. 1994, 98, 7.

(22) Fredrickson, G. H.; Helfand, E. J. Chem. Phys. 1987, 87, 697.

(23) Costanzo, P. J.; Beyer, F. L. Macromolecules 2007, 40, 3997.

(24) Matsen, M. W.; Bates, F. S. J. Phys. Chem. 1997, 106, 2436.

(25) Reister, E.; Fredrickson, G. H. Macromolecules 2004, 37, 4718.

(26) Semenov, A. N. JETP 1985, 61, 733.

(27) Naughton, J. R.; Matsen, M. W. Macromolecules 2002, 35, 8926.

(28) Kim, J. U.; Matsen, M. W. Manuscript in preparation.

MA7024545 